\title{
SiC MOSFETs soft and hard failure modes: functional analysis and structural characterization
}

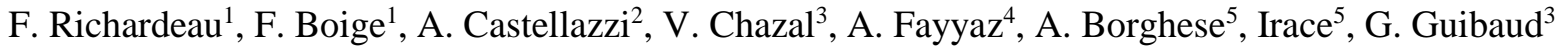 \\ ${ }^{1}$ LAPLACE, University of Toulouse, CNRS, Toulouse INP, UPS, Toulouse, France \\ ${ }^{2}$ Solid-State Power Processing (SP2) Lab, Engineering Faculty, Kyoto University of Adavanced Science, Japan \\ ${ }^{3}$ ITEC Lab, THALES, Toulouse, France \\ ${ }^{4}$ PEMC Group, University of Nottingham, Nottingham, UK \\ ${ }^{5}$ DIETI, University of Naples "Federico II", Italy
}

frederic.richardeau@laplace.univ-tlse.fr: alberto.castellazzi@kuas.ac.jp; andrea.irace@unina.it

\begin{abstract}
This paper discusses first the discriminating phenomena yielding either a fail-to-short or fail-to-open circuit signature in $1200 \mathrm{~V} \mathrm{SiC}$ power MOSFETs undergoing shortcircuit electro-thermal stress. Since fail-to-open behavior is of particular relevance to the application, the paper goes on to propose a benchmarking of a number of commercial devices, identifying a single product which offers consistent fail-to-open characteristics with bias voltages up to at least $50 \%$ of nominal rating. For that particular device, a thorough functional and structural characterization is presented. In particular, it is shown that: the gate current is an effective monitor of ensuing degradation under short-circuit stress and can be used to assess damage accumulation, as well as the reversible or permanent nature of device degradation; fail-to-open signature is associated with degradation of the gate-structure, with the creation of shortcircuits between the gate and source terminals in regions relatively far away from the active cells and not involving the field oxide. The findings are relevant to application of both discrete devices and multi-chip power modules, including multiple parallel connected dies.
\end{abstract}

Keywords-Silicon carbide; SiC MOSFETs; short-circuit robustness; fail-to-open.

\section{INTRODUCTION}

Silicon carbide (SiC) power MOSFETs are rapidly gaining increasing relevance in large market applications, including harsh operation and equally reliability sensitive environments, such as automotive or avionics. A key robustness requirement in such domains, particularly in the case of electric drive applications, is short-circuit (SC) withstand capability. A substantial body of literature has already been produced on the subject, pointing out potential criticalities in this respect, when transitioning from silicon ( $\mathrm{Si}$ ) transistors to $\mathrm{SiC}$ ones (see [1-4], for instance). In particular, the studies in [5] have pointed out the existence of a twofold SC failure mode, depending on the rate of temperature increase: a soft one, characterized by the device failing as an open circuit drain to source (Fail-To-Open behavior, FTO) and a hard one, in which the transistor fails as a short-circuit drain to source (Fail-To-Short behavior, FTS). The two different behaviors are exemplified in the results of Fig. 1 a): the former features a collapse of the gate-source
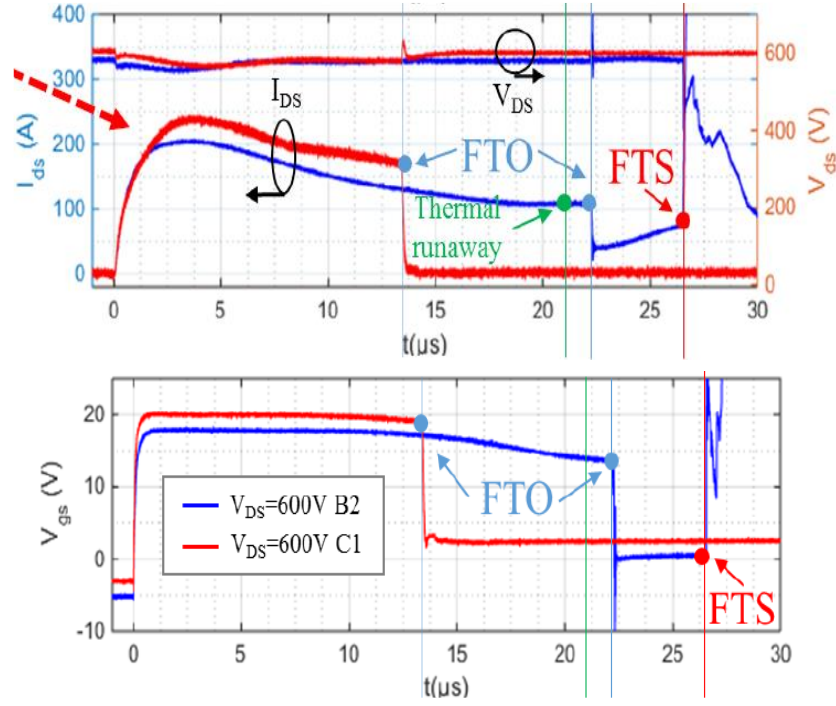

a)

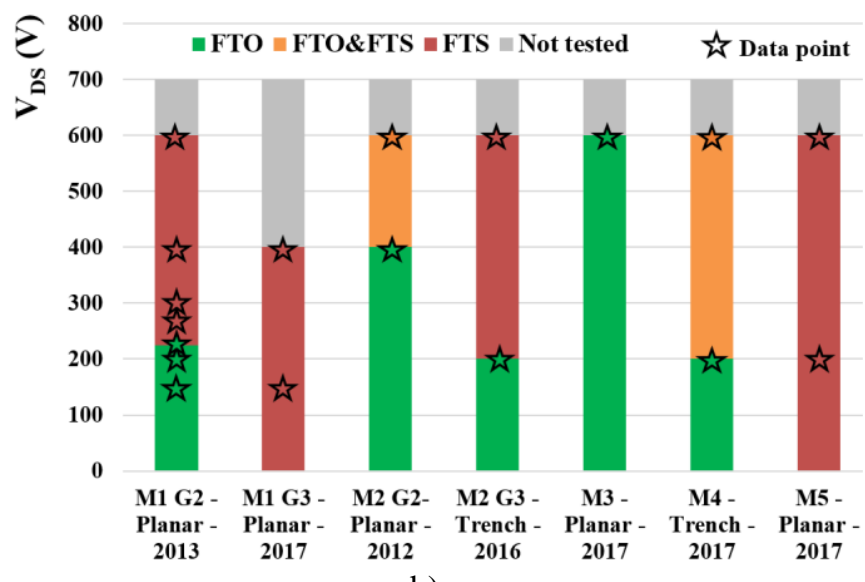

b)

Fig. 1: short-circuit current $I_{D}$ and $V_{D S}$, (top) and $V_{G S}$ (bottom), a); summary of SC failure mode (FTO or FTS) for different commercial devices at different $V_{D S}, \mathrm{~b}$ ). 
voltage to a value well below the threshold voltage (as a result of damage in the gate structure, the gate-source resistance after damage is lower than the external drive gate resistance, $R_{G S}<<$ $R_{G, E X T}$, which practically results in the device turning off the

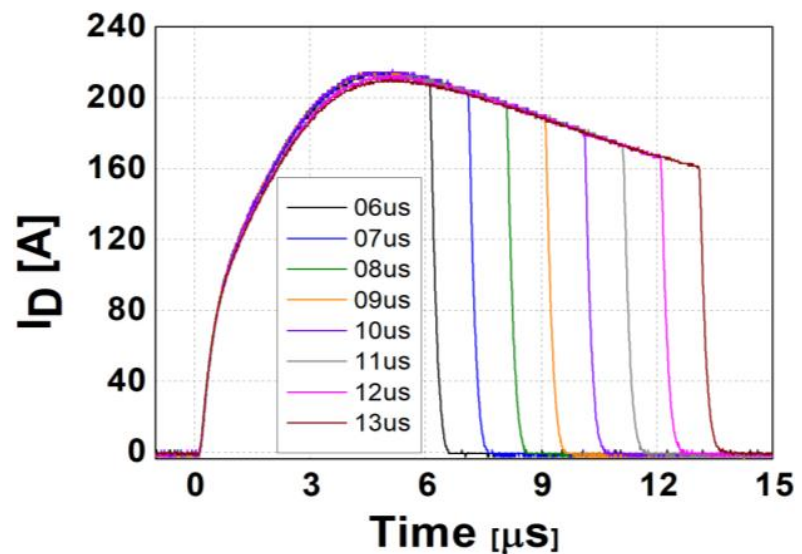

a)

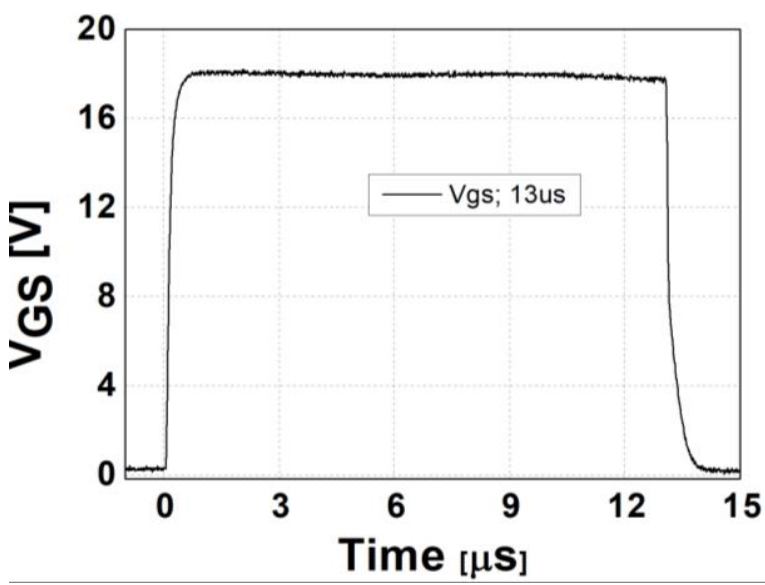

b)

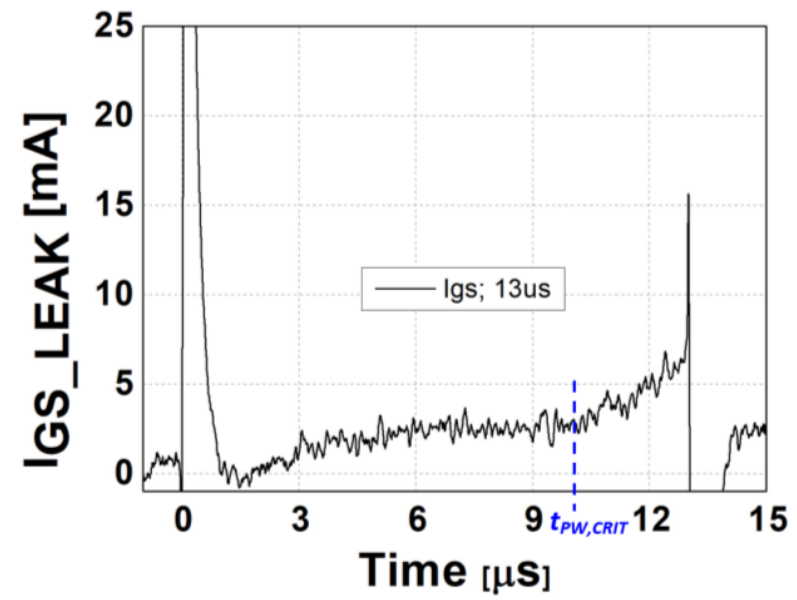

c)

Fig. 2: Experimental results for $\mathrm{SC}$ tests with varying pulse durations: $\left.\left.\left.I_{D}, \mathrm{a}\right) ; V_{G S}, \mathrm{~b}\right) ; I_{G, L E A K}, \mathrm{c}\right)$. A drop in $V_{G S}$ is noticeable (circle) from about $10 \mu \mathrm{s}$, at which moment $I_{G, L E A K}$ features a steeper increase.

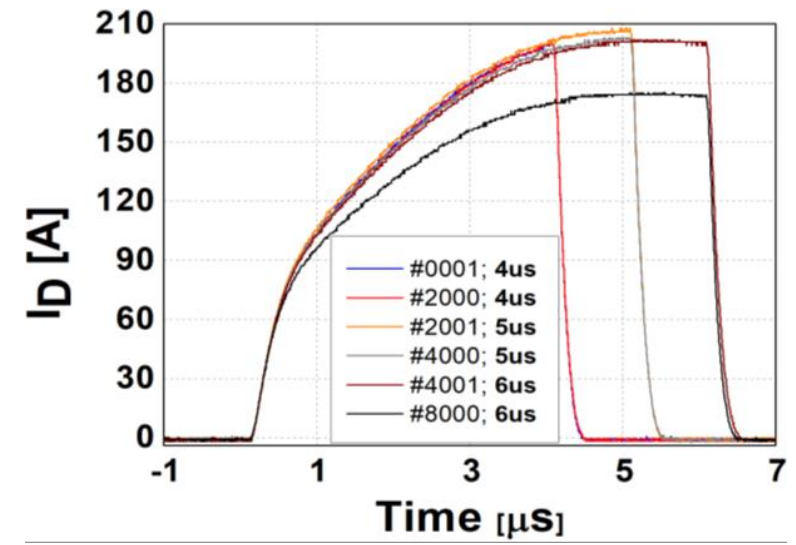

a)

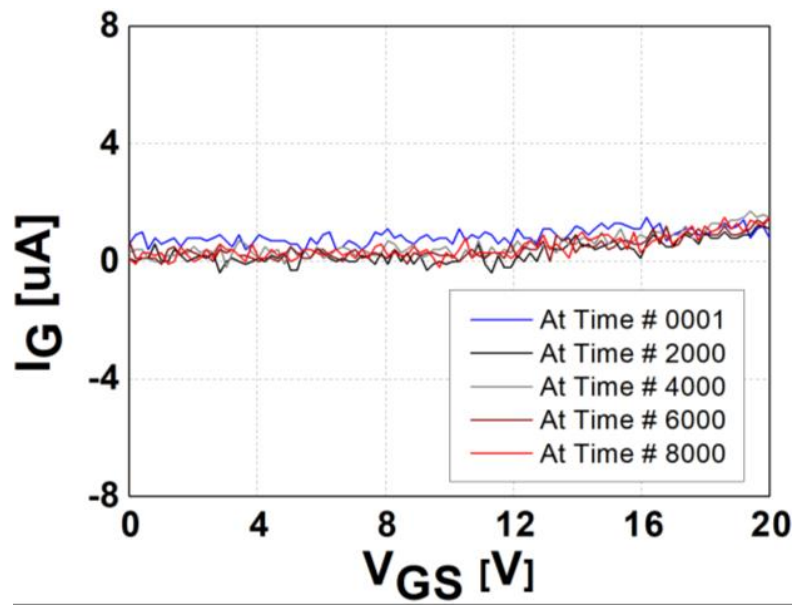

c)

Fig. 3: Experimental results for repetitive SC test with "short" pulse widths: $I_{D}$, a); $I_{G, L E A K}$, b). Though the device characteristics are clearly severely degraded, no relation can be detected with a modification of the gate structure characteristics under these stress conditions.

device; the latter features a shorting of the drain-source terminals, with drain current, $I_{D}$, thermal runaway and collapse of the drain-source voltage, $V_{D S}$. The specific failure mode is of great relevance for the application, with a clear preference for FTO type transistors. Indeed, in contrast to FTO, the FTS mode typically implies: a) the fast discharge of energy storage components (e.g., capacitors), with oftentimes explosion-like characteristics and a possible failure propagation typically for a module device where chips are close to each other ; b) loss of functionality of the whole circuit in the case of parallel multichip architectures, either discrete or module based.

A preliminary screening on commercial $1.2 \mathrm{kV}$-rated MOSFETs delivered the results in Fig. 1 b): today a single manufacturer could be identified, whose device consistently performed as FTO for $V_{D S}$ values up to $50 \%$ rated voltage, which is a representative application requirement [6]. Because of its application interest, that device was considered further in detail. 


\section{DEVICE STRESS AND DEGRADATION MONITORING: FUNCTIONAL CHARACTERIZATION}

The gate leakage current, $I_{G, L E A K}$, is an effective monitor of FTO behavior. Past work reported no damage accumulation and device aging for pulse widths below a minimum value, which could be related to a critical $I_{G, L E A K}$ value [7]. So, in this work, first, tests were carried out to identify such critical pulse-width, $t_{P W, C R I T}$. The results of Fig. 2 show: the SC drain current, $\left.I_{D}, \mathrm{a}\right)$; the gate-source voltage, $\left.\left.V_{G S}, \mathrm{~b}\right) ; I_{G, L E A K}, \mathrm{c}\right)$, for the device subjected to pulses of different duration. Here, $V_{D S}=600 \mathrm{~V}$ in all tests. During the pulse, at about time $=10 \mu \mathrm{s}$, $I_{G, L E A K}$ shows exponential increase onset, which, according to the results presented in [7] and physically analyzed and modeled in [8] as a Schottky emission current, was retained as the $t_{P W, C R I T}$ for the device under test (DUT) here.

The pulse width was then halved and repetitive stress was applied: Fig. 3 a) shows degradation of the saturation characteristics after thousands of pulses; however, that was not found to be related to any degradation at gate level, as the results of Fig. 3 b) clearly shows. On the other hand, increasing the pulse width yields very different results, Fig. 4: the drain current is still degraded, a), but that is now clearly due to damage of the gate-structure, manifest in the form of substantial $I_{G, L E A K}$ value increase, on an accelerated basis between \#3000 and \#3200 cycles , Fig. 4 b), and subsequent decrease, as a result of a low value series gate resistance in the transistor driving path, of the bias voltage applied across the gate-source terminals, Fig. 4 c).

\section{STRUCTURAL CHARACTERIZATION}

The results are interpreted on the basis of two different failure mechanisms intervening, depending on the pulse width: for "short" pulses $\left(<t_{P W, C R I T}\right)$ only reduced saturation current is manifest (Type 1 degradation), possibly caused by degradation of the chip metallization and subsequent $V_{G S}$ de-biasing [2; 9]; for "long" pulses, gate structure aging is evident (Type 2 degradation). The latter is caused by cracks around the interlayer dielectric (ILD) triggered by the high difference in thermal expansion between the top $\mathrm{Al}$ and the $\mathrm{SiO}_{2}$ and formation of metallic paths in the gate-source ILD, caused by the top Al melting at the edge of the chip during the shortcircuit, as presented in Figs. 5 a) and b) [6]. The region of major degradation and thus leading to eventual failure was observed to change on the device surface, but it was always located near the border of the active area, where terminations of various type are present and where geometrical singularity appears between the peripheral gate distribution ring and the interdigital ramifications fingers, Figs. 6 a) and b). However, the failure mechanism remained the same, even in the proximity of active cells, Fig. $6 \mathrm{c}$ ), which can be reasonably assumed to be the locations reaching highest temperatures.

\section{CONCLUSION}

$\mathrm{SiC}$ power MOSFETs exhibit some key differences with $\mathrm{Si}$ MOSFETs and IGBTs. In particular, their features-set implies higher electro-thermal stress levels during short-circuit. On top of that, the present level of technology maturity is still associated with a greater spread in the value of some of the

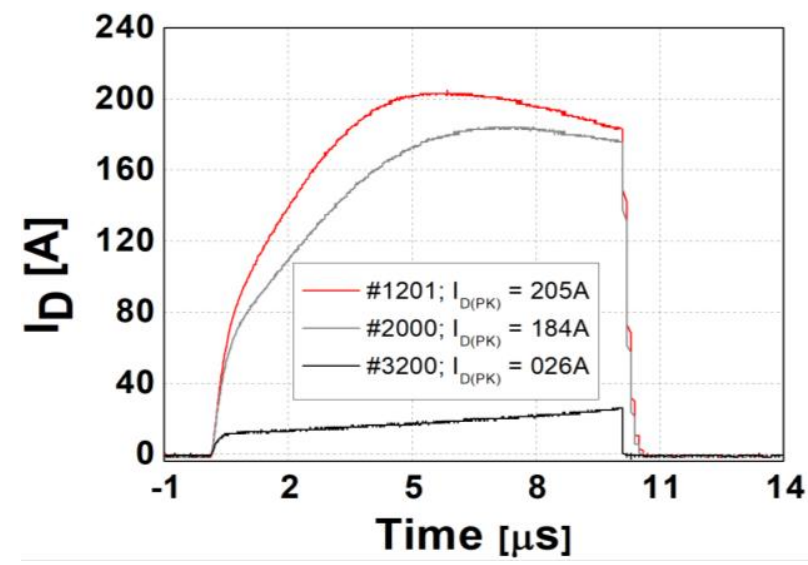

a)

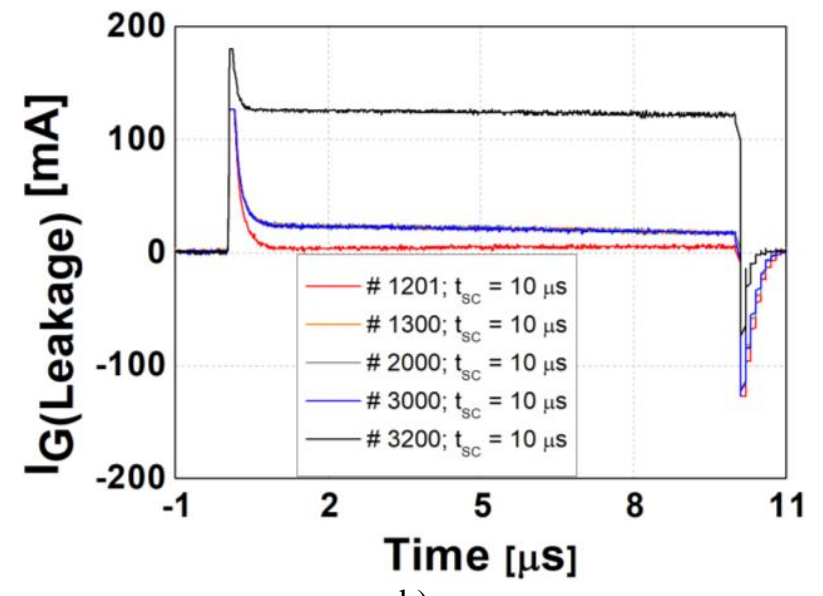

b)

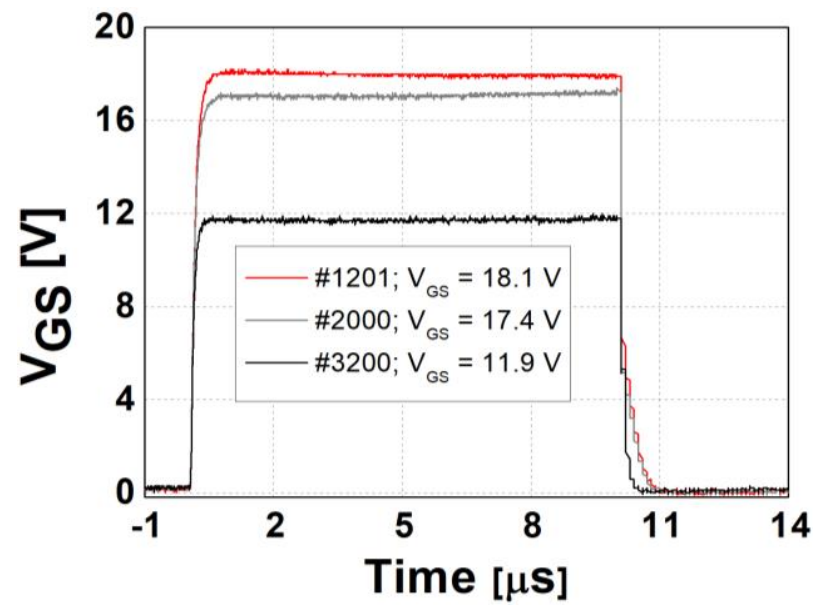

c)

Fig. 4: Experimental results for repetitive SC test with "long" pulse widths: $\left.\left.\left.I_{D}, \mathrm{a}\right) ; V_{G S}, \mathrm{~b}\right) ; I_{G, L E A K}, \mathrm{c}\right)$. In this case, the change in device characteristics is strictly related to degradation of the gate, as manifest by the significant increase in gate current value.

main device electro-thermal parameters, such as threshold voltage and on-state resistance. So, rather uneven degradation in parallel multi-chip structures is a likely occurrence. The 


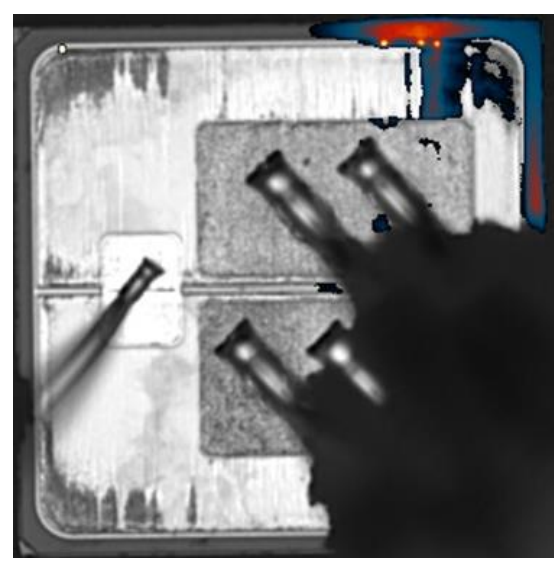

a)

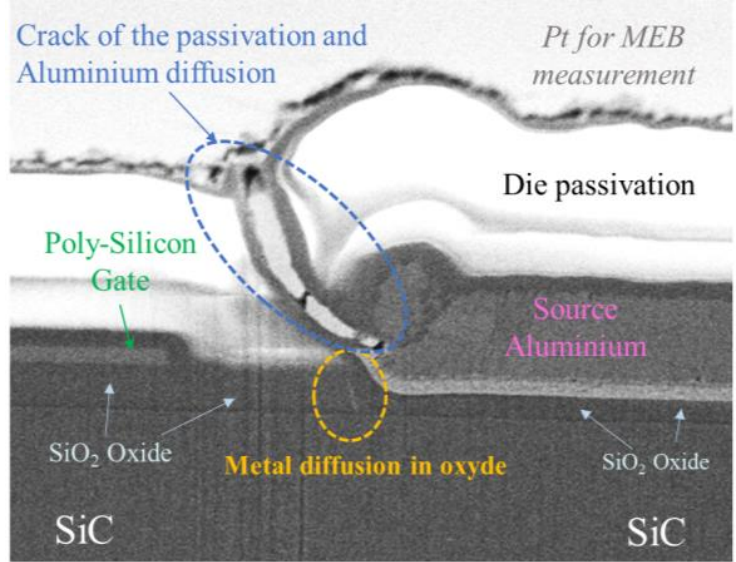

b)

Fig. 5: Micrograph of MOSFET (bare-die top view) showing localized $I_{G, L E A K}$ increase, a); Scanning electron microscope (SEB) image of a device focused ion beam (FIB) microsection after destructive test, b).

ability to ensure a soft (FTO) type of failure ensures the capability to still operate the module, even in the presence of a single die failure, enabling for instance, so-called "homehopping" system level operation mode in critical equipment such as automotive or avionics. Studies have already been undertaken to steer and control the occurrence of the desired FTO event in a single chip, without compromising the controllability of other parallel connected devices $[10,11]$. A permanent gate leakage current of several $\mathrm{mA}$ is a salient precursor, easy to detect and monitor by the gate-driver circuit to reduce ageing and, for instance, trigger a supervisory system to stop operation at a suitable time to replace one or more devices in the system, before any failure occurs.

\section{REFERENCES}

[1] A. Castellazzi, T. Funaki, T. Kimoto, T. Hikihara, Short-circuit tests on SiC power MOSFETs, 2013 IEEE 10th International Conference on Power Electronics and Drive Systems (PEDS), 22-25 April 2013, Kitakyushu, Japan.

[2] A. Castellazzi, A. Fayyaz, L. Yang, M. Riccio, A. Irace, Short-circuit robustness of SiC Power MOSFETs: Experimental analysis, 2014 IEEE
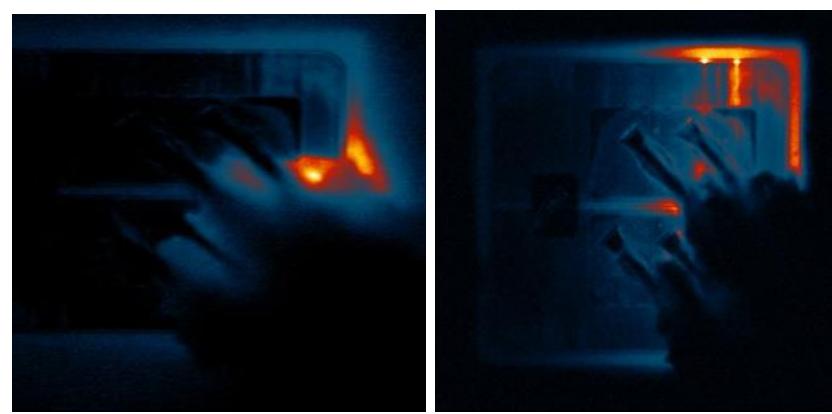

a)

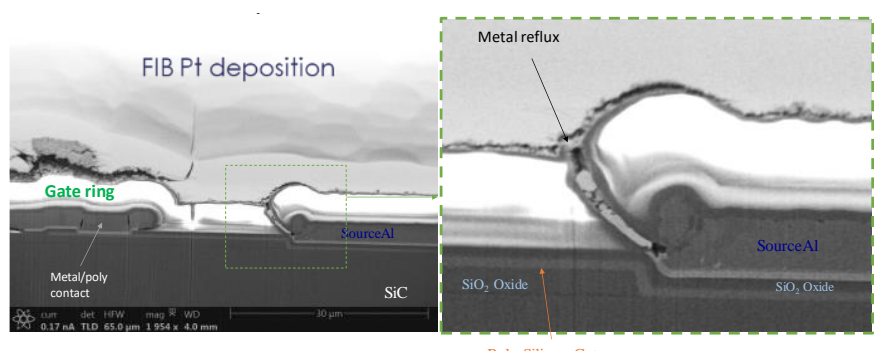

b)

Fig. 6: Micrographs of MOSFET (bare-die top view) showing movement of localized $I_{G, L E A K}$ increase, a); SEB image of device FIB microsection confirming the failure mechanism attribute to $I L D, \mathrm{~b}$ ) (the right hand-side image is a zoom of the area highlighted on the left hand-side one).

26th International Symposium on Power Semiconductor Devices \& IC's (ISPSD), 15-19 June 2014, Waikoloa, HI, USA.

[3] A. Castellazzi et al., SiC power MOSFETs performance, robustness and technology maturity, Microelectronics Reliability Volume 58, March 2016, Pages 164-176

[4] G. Romano et al., Short-circuit failure mechanism of SiC power MOSFETs, in Proc. 2015 IEEE 27th International Symposium on Power Semiconductor Devices \& IC's (ISPSD), 10-14 May 2015, Hong Kong, China.

[5] G. Romano et al., A Comprehensive Study of Short-Circuit Ruggedness of Silicon Carbide Power MOSFETs, IEEE Journal of Emerging and Selected Topics in Power Electronics, Vol. 4, N. 3, Sep. 2016, pp. $978-$ 987.

[6] F. Boige et al., Ensure an original and safe "Fail-to-Open" mode in SiC MOSFET devices in extreme short-circuit operation, Microelectronics Reliability (88-90) 2018, 598-603.

[7] C. Chen et al., Study of short-circuit robustness of SiC MOSFETs, analysis of the failure modes and comparison with BJTs, Microelectronics Reliability 55 (2015) 1708-1713

[8] F. Boige et al., Physical Origin of the Gate Current Surge During ShortCircuit Operation of SiC MOSFET, IEEE Electron Device Letters, vol. 40, no. 5, pp. 666-669, May 2019. doi: 10.1109/LED.2019.2896939

[9] V. Smet et al., Ageing and Failure Modes of IGBT Modules in HighTemperature Power Cycling, IEEE Trans. on Ind. Electr., vol. 58, no. 10, pp. 4931-4941, Oct. 2011.

[10] F. Boige, A. Fayyaz, A. Castellazzi, F. Richardeau, S. Vinnac, Shortcircuit robustness of parallel SiC MOSFETs andfail-safe mode strategy, European Power Elecrtonics and Applications 2019 - IEEE ECCE Europe, Sep 2019, Genoa, Italy.

[11] A. Castellazzi, A. Fayyaz, R. Kraus, SiC MOSFET Device Parameter Spread and Ruggedness of Parallel Multichip Structures, Materials Science Forum, 2018. 